\title{
Distributed Power Control in Wireless Communication Systems
}

\author{
S. Jagannathan \\ Dept of Electrical and Computer Engg, \\ The University of Missouri-Rolla, \\ Rolla, Missouri 65401 \\ sarangap@umr.edu
}

\author{
A. T. Chronopoulos, S. Ponipireddy \\ Computer Science Dept, \\ University of Texas at San Antonio, \\ San Antonio, TX 78249 \\ \{atc,sponipir\}@cs.utsa.edu
}

\begin{abstract}
Energy efficiency is a measure of performance in wireless networks. Therefore, controlling the transmitter power at a given node increases not only the operating life of the battery but also the overall system capacity by successfully admitting new links between a source and a destination. It is essential to find effective means of power control of point-to-point, broadcasting and multicasting scenarios. The wireless networking presents formidable challenges and we consider the problem of unicast or point to point (peer-to-peer) communication in wireless networks in the presence of other nodes. We study the feasibility of admitting new links in an operating area in a wireless network while maintaining the quality of service $(\mathrm{QoS})$ in terms of Signal to Interference ratio (SIR) for each link. The SIR is maintained by adjusting the transmitter power levels at each source for a given link. Distributed power control (DPC) is a natural choice for such purposes because, unlike centralized power control, DPC should be able to adjust the power levels of each transmitted signal using local measurements, so that in a reasonable time, all nodes/links will maintain the desired SIR. In this paper, we present a suite of DPC schemes using both the state space and optimal control methodology in discrete-time. Further, we prove the convergence of the overall network with our algorithm using Lyapunov stability analysis in comparison with an available DPC scheme in the literature. We present simulation results and comparisons to one of the well known DPC scheme in the literature for point to point communications in the case of overlapping scenario.
\end{abstract}

Key words and Phases: Signal to interference ratio (SIR), Energy efficiency, Wireless Network, Distributed Power Control.

\section{INTRODUCTION}

Our approach to energy efficient communication departs from the traditional layered structure in that we jointly address the issue of transmitted power levels in point to point communication scenario via energy efficient approach, similar to that of [1]. As indicated in [1], such joint decisions on connectivity and routing can result in significant improvement in energy efficiency, as compared to a rigid layered structure that makes these decisions independently. Further, our approach is based on link-based nature.

The focus is on source-initiated point to point multiple access system of session traffic. Here, our objective is to construct a minimum energy point to point link, routed at the source, that reaches a desired destination. A new link-based algorithm that takes into account wireless network properties is presented. A crucial issue in wireless networks is the trade-offs between the reach of wireless transmission and resulting inteference by that transmission. We assume that the power level of transmission can be chosen within a given range of values. Therefore there is a tradeoff between reaching a node in a single hop by using higher power (but at the cost of higher interference) versus dropping that link that generates a very high interference to others.

In any point to point multiple-access system, the need for power control is evident. The problems of point to point multiple-access in all-wireless networks are being currently studied. The wireless networking environment presents formidable challenges to the above study. Among the most difficult issues related to mobile wireless applications is that of operation in limited-energy environments. In this paper we propose a distributed power control scheme with one of the main Quality of Service feature that considers 'SIR' for the addition of each link. The scheme developed in [1] addresses the issues of transmitter power levels (and hence network connectivity), and the formation of a link (routing). This approach relies on the 'link-based' nature of wireless communication.

To assess each complex trade-off separately, we assume the following:

- No mobility.

- The availability of a large number of bandwidth resources. (So that contention for channel is not an issue)

- Sufficient transceiver resources are available at each node. (So calls are never blocked)

- The channel conditions are unchanged.

Under these assumptions we focus on the determination of minimum-energy point to point link construction taking into consideration the SIR. In the following sections we study the wireless communication model (Section 2), distributed power control (Section 3), state space-based control design (Section 4), optimal control design (Section 5), simulations (Section 6) and conclusions (Section 7).

\section{A Wireless COMMUNiCATiOnS Model}

In wireless networks it is possible to establish a link between any pair of nodes, provided that each has a transceiver available for this purpose and that the SIR at the receiver node is sufficiently high, (i.e above the required threshold). The links in the wireless networks are determined depending on factors such as distance between nodes, transmitter power, error-control 
schemes, other-user interference, and background noise. Furthermore, in wireless networks no distinction can be made between uplink and downlink traffic. This complicates the interference environment.

The connectivity of the network depends on the transmission power and the interference at the receiver node. Assuming that each node can choose its own power level, which is not to exceed a maximum value $P_{\max }$. We assume that the receiver signal power varies as $r^{-\alpha}$, where $r$ is the range and $\alpha$ is a parameter that typically takes on a value between 2 and 4 depending on the characteristics of the communication medium.

\section{Distributed Power Control (DPC)}

Our goal is to maintain a required SIR threshold for each network link while the transmitter power is adjusted so that the least possible power is consumed. Suppose there are $n$ links in the network. Let $G_{i j}$ be the power loss(gain) from the transmitter of the $i$ th link to the receiver of the $j$ th link. It involves the free space loss, multi-path fading, shadowing, and other radio wave propagation effects, as well as the spreading/processing gain of CDMA transmissions [7]. The power attenuation is taken to follow the inverse fourth power law

$$
G_{i i}=\frac{g}{r_{i j}^{\alpha}}
$$

Calculation of SIR $R_{i}$ at $i$ th link, [3]

$$
R_{i}=\frac{G_{i i} * P_{i}}{\left(\Sigma_{j \neq i} G_{i j} * P_{j}+\eta_{i}\right)},
$$

where $i, j \in\{1,2,3, \ldots, n\}, P_{i}$ is the $i^{\text {th }}$ link's transmitter power and $\eta_{i}>0$ is the thermal noise at its receiver node. For each link $i$ there is a lower SIR threshold $\gamma$. We take the threshold to be the same as $\gamma$ for all links, reflecting a certain QoS the link has to maintain in order to operate properly. Therefore, we require

$$
R_{i} \geq \gamma
$$

for every $i=1,2,3, \ldots, n$. An upper SIR limit is also set, so that the transmitter power of a link is minimized, which in turn will decrease the interference due to its transmitter power at the other receiver nodes. Therefore, we check:

$$
R_{i} \leq \gamma^{*}
$$

The above condition is used to minimize the transmitter power but it is not mandatory. If the equation (3) or (4) fails (i.e $R_{i}<\gamma$ ) then the transmitter power of the transmitter has to be updated using equation (5). Therefore each link independently increases its power when its current SIR is below its target $\gamma$, and decreases it otherwise. The associated power update can be obtained from [4] as

$$
P_{i}(k+1)=\frac{\gamma P_{i}(k)}{R_{i}(k)}
$$

where $k=(1,2,3, .$.$) (see [3] - [5]). If P_{i}(k+1)>P_{\max }$, the new link is not added. If the power slips under the minimum threshold power $P_{i}(k+1)<P_{\min }$ (the minimum power needed to form a link), then we keep $P_{i}(k+1)=P_{\text {min }}$.

\section{SIR protection of an active link}

For any active link $i$, that we have $R_{i}(k) \geq \gamma \quad=>R_{i}(k+$ 1) $\geq \gamma$.

This implies that a new link is added if and only if the new state of the system is stable, i.e. none of the existing links are broken.

We next present the distributed algorithm with power control. Algorithm

1. The SIR of the first link is calculated which includes only thermal noise, and it is added.

2. While there are more new links, each new link is started with $P_{\min }$ as the minimum power:

a. All links recalculate its SIR and checks against the threshold.

b. If any of the links transmitter power exceeds $P_{\max }$ then, ignore that link and goto step 2 .

c. If any of the conditions given in (3) or (4) is not satisfied then the new transmitter powers are calculated according to the equations (5), and do part (a).

The final state contains all the feasible links added out of $n$ links maintaining a stable state. Though the work of [4] can be expressed in the above algorithm, but there is strong evidence that the power update can cause convergence problems. First of all, there are no analytical results showing that the algorithm in [4] converges both for overlapping and nonoverlapping cases. Second, results are not included to demonstrate their conclusions with regards to speed of convergence. Our work as presented in this paper is towards addressing the above limitations. A suite of closed-loop distributed power control schemes are presented next. We propose both a state space and optimal control scheme for updating the transmitter power and using Lyapunov stability theory, the convergence proofs are detailed.

\section{State Space-Based Control Design (SSCD)}

Using state space theory [8] our goal is to maintain a required SIR threshold for each network link while the transmitter power is adjusted so that the least possible power is consumed. Using (2) the SIR denoted here as, $R_{i}$, at the $(k+1)$ th iteration can be written as

$$
R_{i}(k+l)=R_{i}(k)+v_{i}(k),
$$

where by defnition $R_{i}=P_{i} / I_{i}$, and interference $I_{i}(k)=$ $\left(\sum_{j \neq i}^{n} P_{j} * \frac{G_{i j}}{G_{i i}}+\frac{\eta_{i}}{G_{i i}}\right)$, with $\mathrm{n}$ is the number of active links. The $v_{i}$ in each system should only depend on the total interference produced by the other users. To maintain the SIR of each link above a desired target and to eliminate any steady-state errors [6], a new state is added to the system. This is the integrator of the error, $e_{i}(k)=R_{i}(k)-\gamma$, which is the summation of the previous error values and $\gamma$ is the target SIR value for the link 'i' . Therefore, defining a new state as $\delta(k)$, where

$$
\delta_{i}(k+1)=\delta_{i}(k)+e_{i}(k) .
$$

Let $x_{i}(k)$ for a node is defined as 


$$
x_{i}(k)=\left[\begin{array}{c}
\delta_{i}(k) \\
e_{i}(k)
\end{array}\right],
$$

Then we have

$$
x_{i}(k+1)=\left[\begin{array}{c}
\delta_{i}(k+1) \\
e_{i}(k+1)
\end{array}\right]=A_{i} x_{i}(k)+B_{i} v_{i}(k),
$$

where

$$
A_{i}=\left[\begin{array}{ll}
1 & 1 \\
0 & 1
\end{array}\right] \text { and } B_{i}=\left[\begin{array}{l}
0 \\
1
\end{array}\right]
$$

Theorem 1: Given the DPC as (9), and if the feedback is chosen as $v_{i}(k)=-\left[\begin{array}{ll}k_{1} & k_{2}\end{array}\right] x_{i}(k)$ with $k_{1}$ and $k_{2}$ represent the feedback gains, then the overall system is stable and the actual SIR values converge to their targets for each link.

Proof: Using (9) and the control input, the closed loop dynamics can be written as follows.

$$
x_{i}(k+1)=A x_{i}(k)
$$

where,

$$
A=\left[\begin{array}{cc}
1 & 1 \\
-k_{1} & 1-k_{2}
\end{array}\right]
$$

And the condition $\mid$ eigenvalues $(A) \mid<1$ must be satisfied. To find the eigenvalues, we solve $\operatorname{det}(z I-A)=0$, i.e

$$
\left|\begin{array}{cc}
z-1 & -1 \\
k_{1} & z-1+k_{2}
\end{array}\right|=0
$$

i.e $(z-1)\left(z-1+k_{2}\right)+k_{1}=0$. We assume $\left(k_{2}^{2}-4 k_{1}\right)<0$ to have a pair of complex eigenvalues $z, \bar{z}$. Then the complex eigenvalues have $|z|^{2}=1+k_{1}-k_{2}$. Thus the condition $|z|^{2}<$ $\alpha<1$ gives $|z|<1$. So it suffices to have $k_{1}-k_{2}<\alpha-1$. The values satisfy this condition. These are the feedback control values. If the choosen values are appropriate then the system corresponding to equation (9) will be asymptotically stable.

We chose $k_{1}=1 / 3$ and $k_{1}=1 / 2$. The above values are used to calculate $v_{i}$ i.e.,

$$
v_{i}=-k_{1} \delta_{i}-k_{2} e_{i}
$$

The new power can be computed as follows,

$$
P_{i}(k+1)=R_{i}(k+1) I_{i}(k) .
$$

Using this new power, the SIR's are calculated and checked till the stable state of all the links are achieved. We now consider another algorithm using the state equation for SIR in (9). We select

$$
v_{i}(k)=K_{i} e_{i}(k)-e_{i}(k)
$$

Substituting the above equation in second part of (9) results in

$$
e_{i}(k+1)=K_{i} e_{i}(k),
$$

where $K_{i}$ are the feedback gains to be determined for the closed-loop system.

Theorem 2: Given the hypothesis above, for the system (9), with power control input as in equation (15). Then the closed loop system as given by (16) is asymptotically stable provided that

$$
\left|K_{i}^{T} K_{i}\right|<1
$$

Proof: We select a Lyapunov function,

$$
V=1 / 2 e_{i}^{T} e_{i},
$$

whose first difference is given by

$$
\Delta V=e_{i}^{T}(k+1) e_{i}(k+1)-e_{i}(k)^{T} e_{i}(k)
$$

Substituting (16) into (18) results in

$$
\begin{gathered}
\left(K_{i} e_{i}\right)^{T}\left(K_{i} e_{i}\right)-e_{i}^{T} e_{i}=e_{i}^{T} K_{i}^{T} K_{i} e_{i}-e_{i}^{T} e_{i} \\
=-e_{i}^{T}\left(I-K_{i}^{T} K_{i}\right) e_{i}
\end{gathered}
$$

which is less than zero if (17) is satisfied. This shows that the closed loop system is asymptotically stable. This further implies that the actual SIR values for each link will converge to their respective targets.

We next present an optimal control scheme for admitting more links.

\section{Optimal Control Design}

Given the system described by (9), we choose the output $y_{i}=C x_{i}(k)$, for $i=1, \ldots, n, \mathrm{C}$ is the output matrix and with performance index

$$
J_{i}=1 / 2 \sum_{k=k_{0}}^{\infty}\left[x_{i}^{T} Q_{i} x_{i}(k)+v_{i}^{T} R_{i} v_{i}(k)\right],
$$

where $Q_{i}$ and $R_{i}$ are positive definite real matrices selected by the designer.

Theorem 3: Given the hypothesis presented in the previous theorem for DPC, with the feedback selected as $v_{i}(k)=$ $-K x_{i}(k)$, where the feedback gains are taken as

$$
K=\left(B_{i}^{T} S_{\infty} B_{i}+R_{i}\right)^{-1} B_{i}^{T} S_{\infty} A_{i}
$$

and $S_{\infty}$ is the unique positive definite solution of the Algebraic Ricatti Equation (ARE)

$$
S_{i}=A_{i}^{T}\left[S_{i}-S_{i} B_{i}\left(B_{i}^{T} S_{i} B_{i}+R_{i}\right)^{-1} B_{i}^{T} S_{i}\right] A+Q_{i}
$$

Then the resulting time invariant closed loop system described by

$$
x_{i}(k+1)=\left(A_{i}-B_{i} K_{i}\right) x_{i}(k)
$$

is asymptotically stable.

Proof: See [8].

\section{Simulations}

The setup for the simulation experiments to investigate DPC schemes developed in [4] and our SSCD is as follows. The network region is assumed to span a square region of side 500units as shown in Figure 1. For simplicity, the links are placed uniformly and horizontally, all of equal lengths. Random links are generated for later simulations. All links have the same SIR target $\gamma=5$, which may be low for certain applications. The 
normalized noise floor $\eta_{i} / g$ from equation (2) is taken to be the same for all receivers and equal to $10^{-9}$. The initial power value for each link is assigned as $P_{0}=10^{-5}$ units. We have an upper limit on the power of each link transmitter i.e. $P_{\max }=5$ units. When the transmitter power of an active link is in danger of exceeding $P_{\max }$, while a new inactive link is being admitted, the new link is not added to the system. A new link is added only if it maintains the system's stable state even after its addition. The SIR's of all the active links are maintained just above the target $\gamma$ when the power control scheme given in [4] and ours were implemented for comparison. The admission of a new active link is attempted for a few iterations before the system reaches a stable state. A new link is admitted only if the system still remains in the stable state. If the link is not added then it will be tried later on.

In the other scenario, we took one overlaped link with all other non-overlapped links as shown in Figure 1.Links are added and made active as their SIR's are above the target value. If a link wants to terminate, its transmitter power is made zero, so that it does not cause any interference to other active links. Here we can see that the overlaped link was not added when the power control scheme in [4] was used in Figure 2, while it was successfully added using our SSCD scheme (see Figure 3) in less number of iterations. Further, in all the scenarios, the speed of convergence is faster in comparison with the others. This clearly shows the superiority of our DPC scheme over the one presented in [4]. In another study, the total power of the system is observed to be changing with the amount of overlapping. In other words, overlapping effects the total power of the entire sytem (sum of all the transmitter powers) and the probability of admission of new links. More overlapping results in higher transmitter powers and fewer new link admissions and vice versa. Here also, our DPC scheme provides a superior performance in terms of increasing the system capacity by simultaneously admitting several new links.

\section{CONCLUSIONS}

This paper presents a energy efficient power control scheme for wireless networks. Though a point to point communication link is considered in this paper, but the proposed scheme is even applicable for constructing energy efficient broadcasting and multicasting trees. Using a novel power update, it is shown that the overall system maintains a desired target SIR value for each link analytically. The analytical results are verified experimentally in two scenarious of overlapping and nonoverlapping. A suite of power control schemes are proposed based on state space control, design and analysis and optimal control methodology. Preliminary results show that our state space approach offers a superior performance in terms of convergence compared to the available ones in the literature.

\section{ACKNOWLEDGMENTS}

This research was supported, in part, by research grants from 1) NSF Grant ECS\#0269161, 2) University of Missouri Research Board Award (3) NASA NAG 2-1383 (1999-2001), (4) State of Texas Higher Education Coordinating Board through the Texas Advanced Research/Advanced Technology Program ATP 003658-0442-1999

\section{REFERENCES}

[1] A. Ephremides, J. E. Wieselthier and G. D. Nguyen, "On the Construction of Energy-Efficient Broadcast and Multicast Trees in Wireless Networks". pp.585-594, IEEE INFOCOM 2000.

[2] M. H. Ammar, G. C. Polyzos and S. K. Tripathi, "Special Issue on network support for multipoint communication" IEEE Journal on Selected Areas in Communications, 15, April 1997.

[3] N. Bambos, "Towards Power-Sensitive Network Architectures in Wireless Communications:concepts, issues and Design Aspects". IEEE Personal Communications, pp.50-59, June 1998.

[4] N. Bambos, S. Chen and G. J. Pottie, "Channel Access Algorithms with Active Link protection for Wireless Communication Networks with Power Control". IEEE ACM Transactions on networking, pp.583-597, October 2000.

[5] G. J. Foschini and Z. Miljanic, "A Simple Distributed autonomous power control algorithm and its convergence". IEEE Tran. Veh. Tech., vol.42, pp.641-646, April 1993.

[6] A. El-Osery, and C. Abdallah "Distributed Power Control in CDMA Cellular Systems", IEEE Antennas and Propagation Magazine, Vol. 42, No.4, August 2000.

[7] T. S. Rappanport, "Wireless Communications, Principles and Practices", book Prentice Hall, 1999.

[8] F.L. Lewis, "Optimal Control”, John Wiley and Sons, 1999.

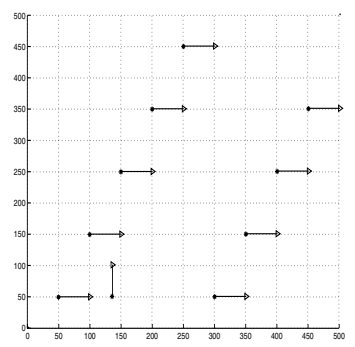

Fig. 1. Uniform distribution of links with 1 overlaped link

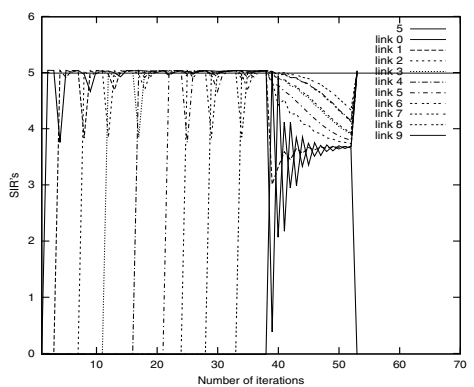

Fig. 2. DPC[4] with 1 overlaped link

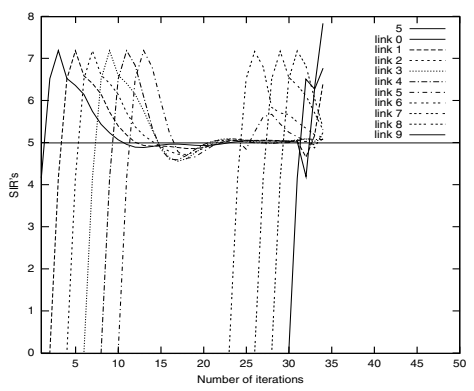

Fig. 3. SSCD with 1 overlaped link 